\title{
Epidemiology of Congenital Heart Defects in Iran: A Systematic Review
}

\author{
Hossein Siabani ${ }^{1}$, Soraya Siabani ${ }^{2 *}$ and Leila Gholizadeh ${ }^{3}$ \\ ${ }^{1}$ Cardiovascular Research Centre, Kermanshah University of Medical Sciences, Kermanshah, Iran \\ ${ }^{2}$ Cardiovascular Research Centre, Kermanshah University of Medical Sciences, Kermanshah, Iran \\ ${ }^{3}$ School of Public Health, University Technology of Sydney, NSW, Australia
}

*Corresponding author: Soraya Siabani, Assistant Professor of health promotion, Kermanshah Cardiovascular Research Center, Kermanshah University of Medical Sciences, Kermanshah, Iran.

Received Date: July 22, 2019

Published Date: August 06, 2019

\begin{abstract}
Background: Congenital heart diseases (CHDs) accounts for more than 30\% of all major congenital anomalies worldwide. Regional factors play an important role in the prevalence and incidence of CHDs. The aim of this systematic review was to summarize the incidence and prevalence of CHDs in Iran
\end{abstract}

Data sources: Medline, EMBASE, Web of Sciences, CINHAL, Google Scholar and PubMed, as well as the Persian (language) databases, plus references listed from relevant articles have been searched to address the research question.

Eligibility criteria: Either original studies or reviews/meta-analysis reporting the prevalence or/and incidence of CHDs among live births in Iran, in which diagnosis of CHD had been based on, at least, physical examination and echocardiography for diagnosing CHDs.

Study appraisal: In this systematic review registered to PROSPERO, the Quality Assessment Tool for Observational Cohort and Cross-Sectional Studies was used to appraise the quality of related studies, and the Preferred Reporting Items for Systematic Review and Meta-Analysis Protocol (PRISMA) was followed for reporting the results.

Result: Among 69 identified publications in English and Farsi, without time restrictions, only 4 studies were eligible to be included in the review. Totally, 114523 live births registered in the referral hospitals, for childbirth in Iran, had been examined for CHDs in which vary prevalence for CHDs were reported from 4 per 1000 in the North East to 8 per 1000 in the Northwest of the country.

Conclusions: high prevalent CHDs in Iran underscore the importance of culturally appropriate prevention and management strategies. Also, a wide range of CHD prevalence in different areas, in Iran, suggests epidemiological investigations, preferably a population-based study with adequate sample size or a study compromised all birth setting.

Keywords: Cardiac defect; Congenital heart disease; Cardiac anomaly; Prevalence; incidence

\section{Introduction}

Congenital cardiac/heart anomalies or congenital heart disease/ defects (CHDs) are important causes of early childhood death and disability [1-3]. These anomalies may involve cardiac valves or/and cardiac internal walls or/and the veins carrying blood from body and lungs to heart or/and the arteries that transport blood from the heart to body and lungs [3]. Diagnosis of CHDs, mostly, is based on physical exam and echocardiography [4]. The cause of CHDs in about $80 \%$ of cases is unknown, even though many are attributable to genetic causes $[1,5]$. CHDs are frequently associated with chromosomal problems (e.g., Down syndrome), and the presence of parental consanguinity and extensive familial aggregation in patients with CHD is also noted [6]. Also, factors such as infections (e.g., rubella), diabetes [7], drugs (e.g., isotretinoin, valproate, and lithium), alcohol and smoking diagnosed as risk factors for CHD [8]. In the same way, regional characteristics probably play an important role in the incidence of CHDs [3].

A wide variety of CHDs, comprising anatomic or hemodynamic lesions, have been diagnosed and coded by the International Classification of Diseases (ICD10) [9]. The American Heart Association has listed the most common CHDs as following: aortic valve stenosis (AVS), atrial septal defect (ASD), coarctation of the aorta (CoA), complete atrioventricular canal defect (CAVC), 
d-transposition of the great arteries, Ebstein's anomaly, hypoplastic left heart syndrome, i-transposition of the great arteries, patent ductus arteriosus (PD), pulmonary atresia, pulmonary valve stenosis, single ventricle defects, tetralogy of Fallot, total anomalous pulmonary venous connection (TAPVC), tricuspid atresia, truncus arteriosus and ventricular septal defect (VSD)[10]. Although many defects are serious, some are minor abnormalities and may resolve without any intervention and some do not manifest until adolescence or adulthood [3].

Cardiac defects, approximately, accounts for 30\% of all major congenital anomalies, and many of cardiac surgery process worldwide. Evidence shows that CHD affects approximately 2-8 per 1000 live births in industrial countries [2,10,11]. However, unfortunately, we know little about CHDs in many countries including Iran.

In Iran, with borders facing Middle Eastern countries, the risk factors are higher which are impacting on a potentially larger incidence of congenital anomalies including CHDs. Specific cultural factors (e.g. consanguineous marriages) and being exposed to hazardous biochemical materials abused during and after the imposed war (1980-1988) [12] are two important predisposing factors. However, there is limited information about the epidemiology of CHD in Iran. It is supposed that the majority of Iranian papers have been published in Farsi language, hence, not available for international English language investigators. Therefore, a systematic review study was conducted aiming to review the published articles critically in order to estimate the prevalence and/or incidence of CHDs among live birth neonates in Iran.

\section{Methods}

A systematic review study was conducted in order to address the research question of "what is the prevalence and incidence of CHDs among live births in Iran? The protocol information of the study that has been registered in the PROSPERO database (register no $=$ CRD42016042854), was developed according to Cochrane collaboration guideline for observational studies [13]. The PRISMA (Preferred Reporting Items for Systematic reviews and MetaAnalyses) was followed for data extraction and analysis [14].

\section{Inclusion criteria}

The criteria for a study to be included in this review were; 1. Reporting the prevalence/incidence of total CHD or/and a CHD subtype, 2. Being an original study (retrospective or perspective) including case controls, cohorts and cross-sectional, as well as systematic reviews or meta-analysis if any, 3 . The results must be published in a peer reviewed journal either in Persian or English, 4. In which the diagnosis of CHD was based on, at least, physical examination and echocardiography, and, fifth, being conducted on the live births in Iran.

\section{Searching literature}

After framing the research question, two investigators, with the assistance of a professional medical librarian, undertook an extensive search for potentially eligible articles published in various medical databases comprising Web of Science (ISI), Medline, PubMed, Scopus, Embase, ScienceDirect, CINAHL, Google Scholar and Iranian medical databases ( ISC , SID, IranDoc, Iran Medex, Magiran, Medlib) containing articles mostly published in Farsi (language) and therefore missed by English language databases. Also, references listed from relevant articles were hand searched for related papers. To identify further information related to the research question, institutional web sites, as well as grey literatures, were reviewed. The primary searches were limited to papers from early 1995 to September 2016; however, due to limited relevant papers the time limitation was removed, and search included all related articles published till November 2016.

\section{Research strategy}

In order to find relevant studies, a logical combination of the keywords of "epidemiology", "incidence", "prevalence", "Iran" and epidemiological research methods and the mesh terms for "congenital heart defect/disease" or one of the most common CHD subtypes listed below were applied to extract related articles. The terms of "Ventricular Septal Defect (VSD)", "atrial septal defect (ASD)", "pulmonary stenosis (PS)", 'patent ductus arteriosus (PDA)", "tetralogy of Fallot (TOF)", "transposition of the great arteries (TGA)", "aortic stenosis (AoS)", "aortic valve stenosis (AVS)", "coarctation of the aorta (CoA)", "complete atrioventricular canal defect", "transposition of the great arteries", "Ebstein's anomaly", "hypoplastic left heart syndrome", "pulmonary atresia", "pulmonary valve stenosis (PS)" and "single ventricle defects" were the subtypes that were used in the combinations. Specific research strategy for each database, if applicable, was used. For example, in order to extract data from the article cited in the Ovid database (Medline) we followed its specific search strategy (Appendix A).

\section{Article selection}

As Figure 1, a PRISMA Flowchart, displays, in the primary searches, 69 papers were retrieved. After excluding the repeated titles and those that obviously were irrelevant, the abstracts of articles were reviewed in order to obtain an overall understanding into the studies and more information regarding study designs and the aim seeking for. In these two steps, 45 out of 67 papers were excluded from our study. The two investigators reviewed the rest articles separately. In-depth review of full texts resulted in selecting 8 articles for quality appraisal, and finally, 4 papers were included in the final analysing Figure 1 and Table 1.

\section{Assessment for risk of bias}

The Quality Assessment Tool for Observational Cohort and Cross-Sectional Studies (QATOCCS) was used [15] to appraise the quality of included studies, critically. Out of 14 items from Cochrane Observational Tool for Observational Systematic Review, five items fitted to our study subjects were; "Was the research question or objective in this paper clearly stated?", "Was the study population clearly specified and defined?", "Was the participation rate of eligible persons at least 50\%?", "Were all the subjects selected or recruited from the same or similar populations (including the same 
time period)?", "Were inclusion and exclusion criteria for being in the study specified and applied uniformly to all participants?". The quality rating used were Good, Fair, or Poor [15] The most important item for us was the Pa Co Co (Participant, Condition, Context) [16] considered for each study in the Reviewing Table 1. Any study that did not meet any of these three criteria was excluded from the review.

\section{Results}

Among 69 papers obtained during the primary searching in the electronic medical databases including English and Farsi, without time limitation, only 4 studies (from 1994-2012) were eligible to be included in this review Table 1 . The rest were excluded due to being irrelevant or/and not answer to the research question appropriately or/and not meet one or more inclusion criterion, or low-quality research method. However, to avoid missing any valuable information, we have reported the results of 3 other studies that were not eligible because of reporting CHD prevalence among both live and stillbirthor no mentioning the diagnostic methods, or poor methodology Table 2 . The included studies had been conducted in the various area across the country where people living with various ethnicity. Totally 60923 live birth babies had been examined by the 4 studies included in this review. Settings were referral hospitals in the different region of Iran with almost similar ethnicity but different culture and languages. One of the studies did not classify subtypes of CHDs in the results. Minimum prevalence (4 per 1000) was reported Mohsenzadeh et al. [17] in western Iran, and Maximum prevalence (16 per 1000) was from Mirzarahimi [18] in the Northwest of Iran Table 1. The three other studies aiming to determine the prevalence of other congenital anomalies (e.g., such as nervous system) or congenital anomalies as a general term rather than specific terms, were retrieved had reported a little information about CHDs. Hence, they have not reported the details of information about the epidemiology of CHDs Table 2.

Table 1: Characteristics of the included papers in the current review study aiming to estimate the prevalence of $\mathrm{CHDs}$ in live births in Iran

\begin{tabular}{|c|c|c|c|c|c|c|c|c|c|c|c|}
\hline $\mathbf{N}$ & $\begin{array}{c}\text { Author / } \\
\text { Ref }\end{array}$ & $\begin{array}{l}\text { context or } \\
\text { specific } \\
\text { setting }\end{array}$ & $\begin{array}{c}\text { Re- } \\
\text { search } \\
\text { method }\end{array}$ & $\begin{array}{l}\text { Condition } \\
\text { (disease) }\end{array}$ & Duration & $\begin{array}{l}\text { Population( } \\
\text { partici- } \\
\text { pants) }\end{array}$ & Sample & Diagnosis & $\begin{array}{l}\text { Preva- } \\
\text { lence / } \\
\text { n/1000 }\end{array}$ & $\begin{array}{l}\text { The most } \\
\text { frequent } \\
\text { subtypes } \\
\text { respectively }\end{array}$ & Note \\
\hline 1 & $\begin{array}{l}\text { Rahim. et } \\
\text { al. (22) }\end{array}$ & $\begin{array}{c}\text { of Ahwaz, } \\
\text { Khuzestan } \\
\text { Province }\end{array}$ & $\begin{array}{l}\text { Retro- } \\
\text { spective }\end{array}$ & $\begin{array}{l}\text { prevalence } \\
\text { of CHDs }\end{array}$ & $\begin{array}{l}1998- \\
2007\end{array}$ & Live Birth & 3061 & $\begin{array}{l}{ }^{*} \mathrm{CE} ; * * \mathrm{EC} ; \\
{ }^{* * *} \mathrm{CD} ;\end{array}$ & 12.3 & $\begin{array}{c}\text { ASD }(19.54 \%) \\
\text { TOF } \\
(16.99 \%), \\
\text { PDA } \\
(17.97 \%), \\
\text { Shunt } \\
\text { (11.47\%) and } \\
\text { VSD }(11.07 \%) .\end{array}$ & \\
\hline 2 & $\begin{array}{l}\text { Nikyar } \\
(2011) \\
(23)\end{array}$ & $\begin{array}{l}\text { Dezyani } \\
\text { hospital in } \\
\text { Gorgan }\end{array}$ & $\begin{array}{l}\text { Retro- } \\
\text { spective }\end{array}$ & $\begin{array}{l}\text { Prevalence } \\
\text { of CHDs }\end{array}$ & $\begin{array}{c}2007 \text { and } \\
2008\end{array}$ & Live Birth & 11739 & $\begin{array}{l}\mathrm{CE} ; \mathrm{EC} ; \mathrm{CD} ; \\
\text { and }{ }^{* * * *} \mathrm{CC}\end{array}$ & 8.6 & $\begin{array}{c}\text { ASD (26\%), } \\
\text { VSD+ASD } \\
(13 \%) \text { and } \\
\text { PDA (13\%) }\end{array}$ & $\begin{array}{l}4.5 \text { and } \\
13.19 \text { per } \\
1000 \text { in } \\
2007 \text { and } \\
2008-(\text { for } \\
\text { male }=9.96 \\
\text { and female } \\
=7.34 \text { ) }\end{array}$ \\
\hline 3 & $\begin{array}{l}\text { Mohsen- } \\
\text { zadeh } \\
\text { et al. } \\
\text { (17) }\end{array}$ & $\begin{array}{l}\text { Khoram } \\
\text { abad Kourd- } \\
\text { ish }\end{array}$ & $\begin{array}{l}\text { Retro- } \\
\text { spective }\end{array}$ & $\begin{array}{l}\text { Prevalence } \\
\text { of CHDs }\end{array}$ & $\begin{array}{l}2006- \\
2011\end{array}$ & Live births & 43195 & CE, EC & 4.09 & $\begin{array}{c}\text { VSD (44\%) } \\
\text { ASD (21\%,) } \\
\text { PS (5.4\%), } \\
\text { ASD+VSD } \\
\text { (5\%) VSD+PS, } \\
\text { PDA, AS, TGA, } \\
\text { VSD+TGA, } \\
\text { SV, TF }\end{array}$ & $\begin{array}{l}\text { Male } 2 \text { times } \\
\text { females }\end{array}$ \\
\hline 4 & $\begin{array}{l}\text { Mirzara- } \\
\text { himi } \\
\text { (18) }\end{array}$ & $\begin{array}{l}\text { Ardabil- } \\
\text { Imam } \\
\text { khomeini } \\
\text { and Alavi } \\
\text { hospitals }\end{array}$ & & $\begin{array}{l}\text { Prevalence } \\
\text { of CHDs }\end{array}$ & $\begin{array}{l}2006 \text { to } \\
2007\end{array}$ & Live Birth & 2928 & CE; EC & 16 & VSD, PDA & $\begin{array}{l}9.9(61.7 \%) \\
\text { female and } \\
6.4(38.3 \%) \\
\text { male }\end{array}$ \\
\hline
\end{tabular}

Table 2: Characteristics of the papers not eligible to be included in the review, but with notable information regarding CHDs epidemiology in Iran

\begin{tabular}{|c|c|c|c|c|c|c|c|c|c|}
\hline $\mathbf{N}$ & Author/Ref & $\begin{array}{c}\text { context or spe- } \\
\text { cific setting }\end{array}$ & $\begin{array}{c}\text { Research } \\
\text { method }\end{array}$ & $\begin{array}{c}\text { Condition } \\
\text { (disease) }\end{array}$ & Duration & $\begin{array}{c}\text { Population } \\
\text { (participants) }\end{array}$ & Sample & Diagnosis & $\begin{array}{c}\text { Prevalence } \\
/ \mathbf{n} / \mathbf{1 0 0 0}\end{array}$ \\
\hline 1 & $\begin{array}{c}\text { Ghahramani et } \\
\text { al. (24) }\end{array}$ & $\begin{array}{c}\text { Gonabad (22 Bah- } \\
\text { man Hospital) }\end{array}$ & Retrospective & $\begin{array}{c}\text { Congenital } \\
\text { anomalies }\end{array}$ & $1994-2001$ & Live births & 12319 & CE, EC & 0.17 \\
\hline 2 & $\begin{array}{c}\text { Mashhadi et } \\
\text { al. (25) }\end{array}$ & $\begin{array}{c}\text { Tabriz, Rural area } \\
\text {-Communty-based }\end{array}$ & Retrospective & $\begin{array}{c}\text { Congenital } \\
\text { anomalies }\end{array}$ & $\begin{array}{c}\text { Between } 2004 \\
\text { and 2012 }\end{array}$ & Live Birth & 22500 & $\begin{array}{c}\text { CE, } * 2 \mathrm{DE} \text { CD, } \\
\text { and CC }\end{array}$ & $1.42 \mathrm{~s}$ \\
\hline 3 & $\begin{array}{c}\text { Ahmadzadeh } \\
\text { et al. (26) }\end{array}$ & $\begin{array}{c}\text { Ahvaz Arvand } \\
\text { Hospital }\end{array}$ & Retrospective & $\begin{array}{c}\text { Congenital } \\
\text { anomalies }\end{array}$ & $2004-2006$ & Live birth & 4660 & CE, EC & 3.2 \\
\hline
\end{tabular}


In conducting the study, we followed PRISMA-P checklist and flowchart [19] We found several appropriate appraisal tools but finally $[20,21]$, In selecting and appraising critically the related studies the Cochrane group tool for the observational study has been used [20].

Table 1 shows the characteristics (such as Author/Reference, context or specific setting, research method, condition (disease), duration, population (participants), sample size, diagnosis, prevalence number per 1000 live birth, the most frequent subtypes respectively) of 4 papers from Rahimi et al. [22], Nikyar [23], Mohsenzadeh et al. [17] and Mirzarahimi [18] which have been included in the final stage of the review study. In these studies, the means for diagnosis CHD had been Clinical examination (CE), and Echocardiography (EC) and/or Color Doppler (CD) and/or Cardiac Catheterization (CC).

Table 2 demonstrates the characteristics of 3 papers that were not be eligible to be included in the final analysis of the review, but with notable information regarding CHDs epidemiology in Iran. They are from Ghahramani et al. [24], Mashhadi et al. [25] and Ahmadzadeh et al. [26]. In these studies, the means for diagnosis CHD had been Clinical examination (CE), and Echocardiography (EC) and/or Color Doppler (CD) and/or Cardiac Catheterization (CC) and/or 2D-echocardiography (2DE).

\section{Discussion}

This study was undertaken to estimate the prevalence and incidence of congenital heart defects (CHD) in Iran. The Finding showed a wide range of CHD prevalence, from 4 per 1000 to 16 per1000, across geographical distributions which are a vast range of prevalence of CHDs in different parts of the country.

The results of the current study indicated that the prevalence of CHDs in Iran is significantly higher than in other nations (4-8 per 1000 live births) $[2,10,11,27,28]$ This huge difference would be more considerable if the studies conducted by Mashhadi et al. [25], Ghahramani et al. [24] and Ahmadzadeh et al. [26] aiming to estimate the prevalence of congenital anomalies rather than focusing on CHDs only, which were excluded due to insufficient information, became included. This big difference could be explained by environmental factors, ethnic origin or cultural factors vary in different parts of Iran. However, the health system researchers are engaged to search inclusively for the reasons behind such a wide range between the prevalence of CHD in different areas.

Regardless of the wide variety in CHD prevalence in different regions of Iran, these results may persuade health policymakers and health care providers to pay more attention to provide prenatal care and adequate consultation before people marriage or getting pregnant. Suggested strategies to prevent CHDs may include vaccination against rubella and other communicable diseases before pregnancy, control of chronic medical conditions (e.g., diabetes), monitoring of medicine with specific side effects (e.g., antiepileptic drugs), encouraging women to avoid being exposed to harmful substances (e.g., some materials used in cleaning and painting) during their pregnancy and, avoid taking herbs, dietary supplements and medicine without consulting their doctors. In addition, stop smoking and drinking alcohol is strongly suggested. Also, taking enough folic acid (400 micrograms daily) from at least one mount before and during pregnancy can prevent some congenital defects including CHDs.

\section{Limitation}

The main limitation for this review was of the low number of high-quality population-based studies illustrating epidemiological aspects of CHD in Iran, therefore, this makes it difficult to estimate prevalence/incidence. In addition, the limited information reported in the included studies may cause difficulty in quality assessment and assessing the risk of bias in each study. This limitation, mainly, for two studies which had reported the prevalence of CHD, just as a part of their main research seeking for whole congenital abnormalities, was significant. Although the included studies met the required inclusion criteria, the quality was variable. Although these data are specific to Iran, these data may provide useful insight into epidemiological characteristics of neighboring countries in the Middle East.

The limited accurate information about the epidemiology of CHDs might be due to various reasons. Poor or insufficient diagnostic facilities, ineffective diseases surveillance system, the failure of researches to be published or published resulted in being unavailable for international researchers might be likely causes.

Denisevan der Linde et al. had conducted a meta-analysis in 2011 to estimate CHD prevalence across the word. They concluded that the information about CHD in the less developed countries is limited [12]. Actually, they have not found any eligible study from Iran. This is not surprising because the studies that were retrieved in our review study were mostly published in Persian databases that are not available for English speakers. Because of a higher rate of predisposing factors such as rubella (German measles) and consanguineous marriages are presented in developing countries [12], despite less information about CHD epidemiology in these regions, the prevalence of CHD likely is higher.

\section{Conclusion}

This review study showed that in spite of studies conducted on different aspects of CHD in Iran, there are limited high-quality studies reporting epidemiological aspects of CHD, Undertaking studies using robust methods, such as the Strengthening the Reporting of Observational Studies in Epidemiology (STROBE) Statement: Guidelines for Reporting Observational Studies Population [29] is needed. In addition, studies, specifically looking for CHD subtypes, are needed to obtain a reliable estimation of not only incidence and prevalence but management strategies in Iran.

\section{Declarations}

\section{Ethical approval}

This article is a systematic review conducted on papers published in the peer-reviewed journal with ethical consideration, hence ethics approval is not applicable here. 


\section{Competing interests}

The authors declare that there they have no conflicts of interest. In addition, the authors have no financial interest related to any aspect of the study.

\section{Acknowledgment}

We would like to thank Mr. Peter Arnold at the University of Sydney for English editing of the manuscript. Also, we greatly appreciate the Kermanshah University of Medical Sciences for funding this project. Also, we thank our willing and helpful participants.

\section{Conflict of Interest}

No conflict of interest.

\section{References}

1. Bruneau BG (2008) The developmental genetics of congenital heart disease. Nature 451(7181): 943-948.

2. Dolk H, Loane M, Garne E (2011) Congenital Heart Defects in Europe: Prevalence and Perinatal Mortality, 2000 to 2005. Circulatio 123: 841849.

3. The National Heart L, and Blood Institute (NHLBI). What Are Congenital Heart Defects? US: NHLBI Health Information Center 2015 [cited 2016].

4. Siabani S, Siabani S, Siabani H, Moeini Arya M, Rezaei F, et al. (2018) Determinants of Adherence to Iron and Folate Supplementation among Pregnant Women in West Iran: A Population-Based Cross-Sectional Study. Quality in Primary Care 25(3): 197-203.

5. Richards AA, Garg V (2010) Genetics of Congenital Heart Disease. Current Cardiology Reviews 6(2): 91-97.

6. Shieh JT, Bittles AH, Hudgins L (2012) Consanguinity and the risk of congenital heart disease. American journal of medical genetics Part A 158A(5): 1236-1241.

7. Wren C, G Birrell, G Hawthorne (2003) Cardiovascular malformations in infants of diabetic mothers. Heart 89(10): 1217-1220.

8. Shi QY, Zhang JB, Mi YQ, Song Y, Ma J, et al. (2014) Congenital heart defects and maternal fever: systematic review and meta-analysis. J Perinatol 34(9): 677-682.

9. American Heart Association (2013) Congenital Cardiovascular Defects. In: American Heart Association editor.

10. Association AH (2016) Congenital heart defects USA 2016.

11. Reller MD, Strickland MJ, Riehle-Colarusso T, Mahle WT, Correa A, et al. (2008) Prevalence of Congenital Heart Defects in Metropolitan Atlanta, 1998-2005. The Journal of pediatrics 153(6): 807-813.

12. Van der Linde D, Konings EE, Slager MA, Witsenburg M, Helbing WA, et al. (2011) Birth prevalence of congenital heart disease worldwide: a systematic review and meta-analysis. Journal of the American College of Cardiology 58(21): 2241-2247.

13. Sanderson S, Tatt ID, Higgins JP (2007) Tools for assessing quality and susceptibility to bias in observational studies in epidemiology: a systematic review and annotated bibliography. International Journal of Epidemiology 36(3): 666-676.
14. Liberati A, Altman DG, Tetzlaff J, Mulrow C, Gøtzsche PC, et al. (2009) The PRISMA statement for reporting systematic reviews and metaanalyses of studies that evaluate healthcare interventions: explanation and elaboration. BMJ 2009 339: b2700.

15. NHLBI (2016) Quality Assessment Tool for Observational Cohort and Cross-Sectional Studies.

16. Harder T (2014) Some notes on critical appraisal of prevalence studies: Comment on: "The development of a critical appraisal tool for use in systematic reviews addressing questions of prevalence". International Journal of Health Policy and Management 3(5): 289-290.

17. Azam Mohsenzadeh, Sasan Saket, Shokofeh Ahmadipour, Babak Baharvand (2014) prevalence and types of congenital heart disease in babies born in the city of Khorramabad (2007- 2011). Yafteh 15(5): 23 29.

18. Mirzarahimi M, Saadati H, Doustkami H, Alipoor R, Isazadehfar K, et al. (2011) Heart Murmur in Neonates: How Often Is It Caused by Congenital Heart Disease? Iranian Journal of Pediatrics. Iran J Pediatr 21(1): 103106.

19. Shamseer L, Moher D, Clarke M, Ghersi D, Liberati A, et al. (2015) Preferred reporting items for systematic review and meta-analysis protocols (PRISMA-P) 2015: elaboration \& explanation. BMJ 350: g7647.

20. Green S, Higgins JPT, Alderson P, Clarke M, Mulrow CD, Oxman AD. 1.2.2. What is a systematic review? In: Higgins JPT, Green S (editors). Cochrane Handbook for Systematic Reviews of Interventions Version 510.

21. Harder T (2014) Some notes on critical appraisal of prevalence studies: Comment on: "The development of a critical appraisal tool for use in systematic reviews addressing questions of prevalence". International Journal of Health Policy and Management 3(5): 289-290.

22. F Rahim, A Ebadi, G Saki, A Remazani (2008) Prevalence of congenital heart disease in Iran: a clinical study. J Med Sci 8(6): 547-552.

23. Nikyar B, Sedehi M, Mirfazeli A, Qorbani M, Golalipour MJ, et al. (2011) Prevalence and Pattern of Congenital Heart Disease among Neonates in Gorgan, Northern Iran (2007-2008). Iranian Journal of Pediatrics 21(3): 307-312.

24. Ghahramani M, Moshki M, Ebadi A (2002) A survey of causes and prevalence of congenital anomalies in live born neonates in Gonabad 22 Bahman Hospital, 1373-80. Ofogh-E-Danesh 1(8): 1-6

25. Mashhadi Abdolahi H, Kargar Maher MH, Afsharnia F, Dastgiri S (2014) Prevalence of Congenital Anomalies: A Community-Based Study in the Northwest of Iran. ISRN Pediatrics.

26. Ali A, Zahad S, Masoumeh A, Azar A (2008) Congenital malformations among live births at Arvand Hospital, Ahwaz, Iran - A prospective study. Pak J Med Sci 24(1): 33-37.

27. Reller MD SM, Riehle-Colarusso T, Mahle WT, Correa A (2008) Prevalence of Congenital Heart Defects in Metropolitan Atlanta, 1998-2005. The Journal of Pediatrics 153(6): 807-813.

28. Vander Linde D KE, Slager MA, Witsenburg M, Helbing WA, Takkenberg JJM, et al. (2011) Birth Prevalence of Congenital Heart Disease Worldwide: A Systematic Review and Meta-Analysis. Journal of the American College of Cardiology 58(21): 2241-2247.

29. Elm Ev, Altma DG, Egge M, Pococ SJ, Gøtzsch PC, et al. (2008) The Strengthening the Reporting of Observational Studies in Epidemiology (STROBE) Statement: Guidelines for Reporting Observational Studies. PLOS 61(4): 344-349. 